\title{
Ergodic-nonergodic transition in a threshold system with feedback
}

$\operatorname{AUTHOR}(S)$ :

Seo, B; Munakata, T

\section{CITATION:}

Seo, B ... [et al]. Ergodic-nonergodic transition in a threshold system with feedback. Physical Review E 2006, 74(2): 026119.

\section{ISSUE DATE:}

2006-08

URL:

http://hdl.handle.net/2433/50312

RIGHT:

Copyright 2006 American Physical Society 
PHYSICAL REVIEW E 74, 026119 (2006)

\title{
Ergodic-nonergodic transition in a threshold system with feedback
}

\author{
Boyoung Seo* and Toyonori Munakata ${ }^{\dagger}$ \\ Department of Applied Mathematics and Physics, Graduate School of Informatics, Kyoto University, Kyoto 606-8501, Japan
}

(Received 26 May 2006; published 28 August 2006)

\begin{abstract}
A threshold system with feedback is studied from the viewpoint of an ergodic-nonergodic transition, a kind of nonequilibrium phase transition, as the rate of input signal variation is changed. By discussing the time evolution of the distribution function, instead of its lowest moment (an order parameter), we can determine the transition point and make clear the role and limitation of the self-consistent equation for the order parameter. Finally the feedback strength is related to an activation energy from a statistical mechanical viewpoint.
\end{abstract}

DOI: 10.1103/PhysRevE.74.026119

PACS number(s): 02.50.-r, 05.10.Gg

\section{INTRODUCTION}

A threshold system is a simple nonlinear system, which is playing important roles in many fields, such as neuroscience [1] and engineering [2] as well as physical science [3,4]. Below we show that this simple system can show an "ergodic-nonergodic" transition when the effects of feedback are taken into account. Ergodic sampling in statistical mechanics and especially in Monte Carlo simulations [10-12] is the one which can sample all of the phase space of the system in a certain (limited) time scale. In this paper ergodic sampling means that the output from the threshold system can follow the (time) variation of the input signal (see Sec. II for a precise definition). With this explanation of the terminology, we proceed to a specification of the system.

Usually we have input signals $s_{n}$ and the corresponding output signals $y_{n}$ with $n=(1,2, \ldots)$ denoting discrete time. For a simple threshold system, the input-output relation is given by [4]

$$
y_{n}=\Theta\left(s_{n}+\xi_{n}-\theta_{n}\right),
$$

where the Heaviside function $\Theta(x)$ is defined by

$$
\Theta(x)= \begin{cases}1 & \text { if } x>0, \\ 0 & \text { otherwise }\end{cases}
$$

In Eq. (1), $\theta_{n}$ is the threshold value and $\xi_{n}$ represents a purely random (white) stochastic process with zero average and a standard deviation $\sigma_{\xi}$. The dynamical behavior of Eq. (1) is rather simple, and it is widely known that the system shows stochastic resonance in which information transfer or the signal-to-noise ratio (SNR), when viewed as a function of noise intensity $\sigma_{\xi}$, has a maximum at nonzero noise intensity [4-6].

In the following we consider that the output signal $y_{n-1}$ is feedbacked $[4,7]$ to the original input signal $s_{n}$ to have

$$
y_{n}=\Theta\left(s_{n}+F y_{n-1}+\xi_{n}-\theta\right),
$$

with $F$ denoting the feedback strength. Comparing Eq. (3) with Eq. (1), we see that the feedback in Eq. (3) is equivalent to Eq. (1) with a time $(n)$-dependent threshold $\theta_{n}$ :

\footnotetext{
*Electronic address: byseo@amp.i.kyoto-u.ac.jp

†Electronic address: munakata@amp.i.kyoto-u.ac.jp
}

$$
\theta_{n}=\theta-F y_{n-1} .
$$

The system (3) was first studied in connection with information transfer when $F$ is very small [4] and there is no hysteresis in the input-output relation. With use of the terminology of the present paper only an ergodic region was considered in [4]. The purpose of this paper is to show an ergodic-nonergodic transition in the system (3) based on the time evolution of the distribution function and clarify the meaning and limitation of a mean-field approach. As a by-product we suggest a relation between feedback strength $F$ and an activation energy.

In Sec. II the time evolution of a distribution function is studied in terms of eigenfunctions and eigenvalues of the transition matrix and the ergodic-nonergodic transition is defined in terms of the eigenvalue $\lambda_{2}$, the second-largest eigenvalue with $\lambda_{1}=1$. In Sec. III we consider two types of timedependent input signals and explicitly illustrate the ergodicnonergodic transition. In Sec. IV the mean-field theory is discussed based on an extended model in which $M$ simple threshold systems are connected in a parallel way. We show that in the limit $M \rightarrow \infty$ the mean-field theory becomes exact. Finally in Sec. V we point out the relation between the feedback strength and an activation energy. This section also contains conclusions of this paper.

\section{TIME EVOLUTION OF A DISTRIBUTION FUNCTION $p_{n}(y)$}

If $\xi_{n}$ in Eq. (3) is a Gaussian white noise with the distribution

$$
p_{\xi}(\xi)=\left(2 \pi \sigma_{\xi}^{2}\right)^{-1 / 2} \exp \left[-\xi^{2} /\left(2 \sigma_{\xi}^{2}\right)\right],
$$

it is seen from Eqs. (3) and (5) that the conditional probability of $y_{n}=1$, when $s_{n}$ and $y_{n-1}$ are given, is

$$
p\left(y_{n}=1 \mid y_{n-1}, s_{n}\right)=(1 / 2) \operatorname{erfc}\left\{\left[\theta-\left(s_{n}+F y_{n-1}\right)\right] /\left(\sqrt{2} \sigma_{\xi}\right)\right\},
$$

with $\quad p\left(y_{n}=0 \mid y_{n-1}, s_{n}\right)=1-p\left(y_{n}=1 \mid y_{n-1}, s_{n}\right) \quad$ where $\operatorname{erfc}(x)=1-\operatorname{erf}(x)$ and $\operatorname{erf}(x)=(2 / \sqrt{\pi}) \int_{0}^{x} \mathrm{~d} u \exp \left(-u^{2}\right)$.

We now consider how the distribution function $p_{n}(y)$ of $y_{n}$ changes with time and define an ergodic-nonergodic transition in the system (3). Our main results are concisely expressed in terms of the time $\tau_{S}$, which denotes the time scale 
for the variation of input signals, thus a large $\tau_{S}$ meaning a slow variation of $s_{n}$. In the plane $\left(F, \tau_{S}\right)$, we find a transition curve $F\left(\tau_{S}\right)$ with $F>(<) F\left(\tau_{S}\right)$ denoting the nonergodic (ergodic) region.

For the time evolution of $p_{n}(y)$, we have an exact equation

$$
p_{n}(y)=\sum_{y^{\prime}=0,1} p\left(y \mid y^{\prime}, s_{n}\right) p_{n-1}\left(y^{\prime}\right) .
$$

Since $y_{n}$ can take two values 0 and 1 , we can also express Eq. (7) by

$$
\boldsymbol{p}_{n}=T\left(s_{n}\right) \boldsymbol{p}_{n-1},
$$

where the $(2 \times 2)$ transition matrix $T\left(s_{n}\right)$ and the twodimensional column vector $\boldsymbol{p}_{n}$ represent $p\left(y_{n} \mid y_{n-1}, s_{n}\right)$ and $p\left(y_{n}\right)$, respectively, by, e.g., $T_{0,1}\left(s_{n}\right)=p\left(y_{n}=0 \mid y_{n-1}=1, s_{n}\right)$ and $\boldsymbol{p}_{n}=\left(\begin{array}{l}p\left(y_{n}=0\right) \\ p\left(y_{n}=1\right)\end{array}\right)$. From Eq. (8) we can also express $\boldsymbol{p}_{n}$ as

$$
\boldsymbol{p}_{n}=T\left(s_{n}\right) T\left(s_{n-1}\right) \cdots T\left(s_{1}\right) \boldsymbol{p}_{0} .
$$

For convenience for later discussions, we calculate the eigenvalues $\lambda_{1}$ and $\lambda_{2}\left(\lambda_{1}>\lambda_{2}\right)$ and the corresponding eigenvectors $\left|\lambda_{1}\right\rangle$ and $\left|\lambda_{2}\right\rangle$, which turn out to be given by

$$
\begin{gathered}
\lambda_{1}=1, \quad\left|\lambda_{1}\right\rangle=\left(\begin{array}{c}
1 \\
\frac{T_{1,0}}{T_{0,1}}
\end{array}\right), \quad\left\langle\lambda_{1}\right|=\frac{T_{0,1}}{T_{1,0}+T_{0,1}}(1,1), \\
\lambda_{2}=T_{0,0}-T_{0,1}, \\
\left|\lambda_{2}\right\rangle=\frac{T_{1,0}}{T_{1,0}+T_{0,1}}\left(\begin{array}{c}
1 \\
-1
\end{array}\right), \quad\left\langle\lambda_{2}\right|=\left(1, \frac{-T_{0,1}}{T_{1,0}}\right),
\end{gathered}
$$

where the eigenvectors are normalized so that $\left\langle\lambda_{1} \mid \lambda_{1}\right\rangle$ $=\left\langle\lambda_{2} \mid \lambda_{2}\right\rangle=1$ and $\left\langle\lambda_{1} \mid \lambda_{2}\right\rangle=0$. From Eq. (10) we see that $-1<\lambda_{2}<1$ or $\left|\lambda_{2}\right|<1$. It is noted that $\lambda_{2}=0$ when $F=0$ and $p_{n}(y)$ depends on only $s_{n}$. This is in contrast with the case $F \neq 0$ where $p_{n}(y)$ depends in principle on signal history $s_{m}(m=n, n-1, \ldots, 1)$; see Eq. (9).

Let us first consider the case where the input signals change very slowly and for some positive integer $K$ we have $s_{m+K} \simeq s_{m}$. Then we may rewrite Eq. (9) as

$$
\boldsymbol{p}_{m+K} \simeq T\left(s_{m}\right)^{K} \boldsymbol{p}_{m}=\left|\lambda_{1}\right\rangle\left\langle\lambda_{1}\left|\boldsymbol{p}_{m}+\lambda_{2}^{K}\right| \lambda_{2}\right\rangle\left\langle\lambda_{2}\right| \boldsymbol{p}_{m} .
$$

If $\left|\lambda_{2}\right|$ is small compared with $\lambda_{1}=1$ and $\left|\lambda_{2}^{K}\right| \ll 1$, we may safely neglect the second term on the right-hand side of Eq. (11) and we have

$$
\boldsymbol{p}_{m+K} \simeq \boldsymbol{p}_{e q}\left(s_{m}\right) \simeq \boldsymbol{p}_{e q}\left(s_{m+K}\right),
$$

where $\boldsymbol{p}_{e q}(s)$ denotes the equilibrium distribution of $y$ when input signal is $s$.

If one defines a relaxation time $\tau_{R}(F)$, which depends on $F$ as the transition matrix $T$ in Eq. (8) itself depends on $F$, by

$$
\tau_{R}(F) \equiv \max _{s}\left\{-1 / \ln \left|\lambda_{2}(s)\right|\right\},
$$

Eq. (12) is expected to be valid as long as the condition

$$
\alpha \tau_{S} \geqslant \tau_{R}(F), \quad \text { or } \tau_{S} \geqslant \tau_{R}(F) / \alpha \equiv \widetilde{\tau}_{S}
$$

is satisfied. Here $\tau_{S}$ is the time scale for variation of input signals and $\alpha$, regarded here as an adjustable parameter, denotes the ratio of the time for variation of a distribution function $\boldsymbol{p}_{n}$ to that of the signal $s_{n}$. We may consider that $\alpha$ does not sensitively depend on the feedback strength $F$ (see below). In Eq. (13) we explicitly show the $s$ dependence of $\lambda_{2}$ and the operation max picks up the value $\tau_{R}$ which maximizes $-1 / \ln \left|\lambda_{2}(s)\right|$ with respect to $s$. Thus as long as the condition (14) is satisfied we may say that the system outputs are able to follow the variation of input signals and this is called an ergodic sampling. When the condition (14) is not met, the system is performing a nonergodic sampling and we would observe a hysteresis as shown below.

We here briefly comment on the case where $\tau_{R}$ becomes large due to $\lambda_{2} \simeq-1$, which actually occurs when $F$ is negative and $|F|$ is large. In this case, however, $\left\langle y_{n}\right\rangle$ does not show any sign of hysteresis as observed in Figs. 1 and 4 (below) due to the rapid oscillation of the distribution $\boldsymbol{p}_{n}$, leading to $\left\langle y_{n}\right\rangle$ which is well represented by an average of the two curves in Fig. 1. From this we omit the case $\lambda_{2} \simeq-1$ from our discussion on the ergodic-nonergodic transition.

\section{ILLUSTRATION OF THE ERGODIC-NONERGODIC TRANSITION}

In order to illustrate the ergodic-nonergodic transition, which we studied from a general viewpoint in the previous section II we consider two types of input signals, one is increasing Eq. (15), and the other decreasing Eq. (16), with time,

$$
\begin{array}{cc}
s_{i n, n}=s_{\min }+\left[\left(s_{\max }-s_{\min }\right) / \tau_{S}\right] n & \left(1 \leqslant n \leqslant \tau_{S}\right), \\
s_{d e, n}=s_{\max }+\left[\left(s_{\min }-s_{\max }\right) / \tau_{S}\right] n & \left(1 \leqslant n \leqslant \tau_{S}\right),
\end{array}
$$

where $s_{\min }$ and $s_{\max }$ denote the minimum and maximum of the input signals and $\left[s_{\max }-s_{\min }\right] / \tau_{S}$ is an input signal velocity. Hereafter in all of our numerical calculations, we set $\theta=1$ in Eq. (3).

In Fig. 1 we show an average $\left\langle y_{n}\right\rangle$ over many experiments [8], with different sequences of noise $\xi_{n}$, as a function of $s$ for $s_{i n, n}$ (solid curve) and $s_{d e, n}$ (dotted curve) for $\tau_{S}=10^{2}$ [Fig. 1(a)], $10^{3}$ [Fig. 1(b)], and $10^{4}$ [Fig. 1(c)] for the case $F=2$ and $\sigma_{\xi}=0.5$. It is observed from Fig. 1 that as $\tau_{S}$ becomes large and the input signals change slowly, the output $y$ loses its hysteresis character and finally becomes a single-valued function of $s$.

To show how one can calculate $\tau_{R}$ from Eq. (13), $-1 / \ln \left|\lambda_{2}(s)\right|$ is plotted in Fig. 2 for $F=2$ and this gives $\tau_{R} \simeq 22$. Similar calculations are performed for each $F$ value, and by setting $\alpha=1 / 25$ we plot in Fig. 3 the curve $\tilde{\tau}_{S}(F) \equiv \tau_{R}(F) / \alpha$. On the other hand, from numerical experiments in which we take various values for $\tau_{S}$, we confirmed that when $\tau_{S}>(<) \tilde{\tau}_{S}$-i.e., in the region above (below) the curve in Fig. 3-an ergodic (nonergodic) behavior is observed. So, when $\tau_{S}$ is fixed, we enter a nonergodic region when $F$ is increased above a critical value $F_{c}\left(\tau_{S}\right)$ at which 

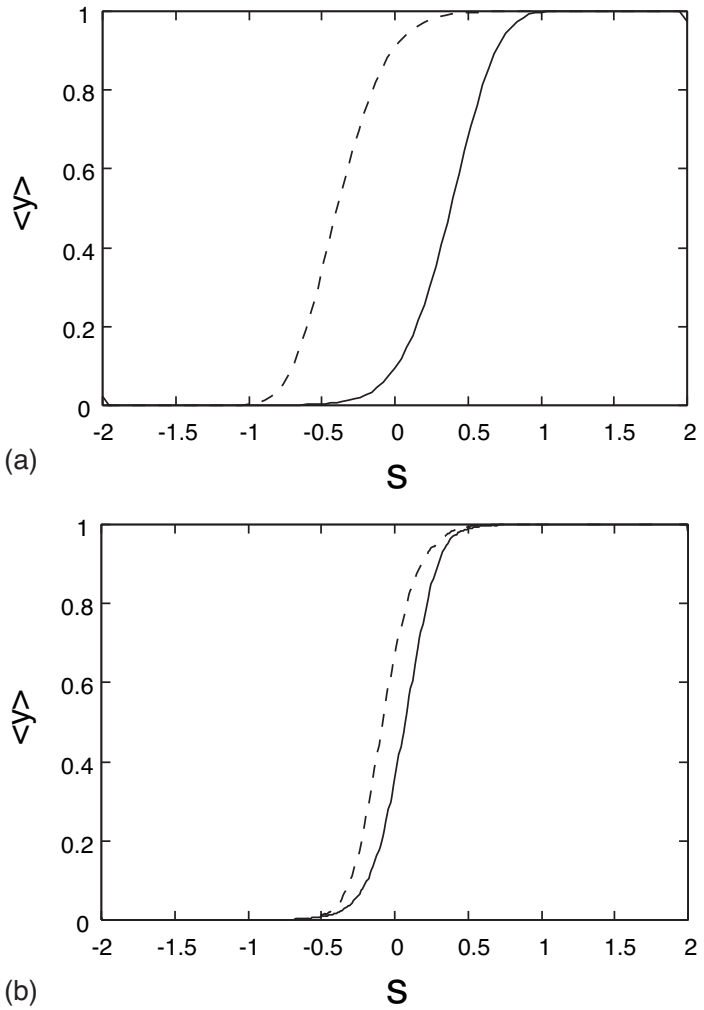

(b)

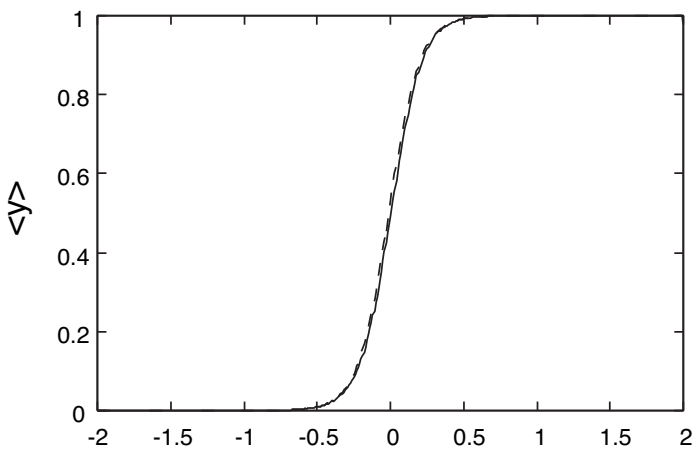

(c)

$\mathrm{S}$

FIG. 1. Variation of $\langle y\rangle$, with $\langle y\rangle$ an average over many $(N)$ experiments, for two types of input signals, Eq. (15) (full curve) and Eq. (16) (dotted curve) with $\tau_{S}=10^{2}$ (a), $10^{3}$ (b), $10^{4}$ (c) $(F=2$ and $\sigma_{\xi}=0.5$ ). As to our numerical experiments with Eqs. (15) and (16), we always choose $N=10^{4}$ and $s_{\min }=-2, s_{\max }=2$.

$\tau_{S}=\widetilde{\tau}_{S}\left(F_{c}\right)$. We note from Fig. 3 that $\widetilde{\tau}_{s}$ increases exponentially for large $F$ and this point is discussed in Sec. V.

The time dependence of $\boldsymbol{p}_{n}$ can be discussed more explicitly based on Eqs. (8) and (10), from which we have

$$
\begin{aligned}
\boldsymbol{p}_{n}= & {\left[T_{0,1} T_{1,0}\right]^{-1}\left\{\left(\begin{array}{l}
T_{0,1} \\
T_{1,0}
\end{array}\right)+\left(T_{0,0}-T_{0,1}\right) T_{1,0}\left[p_{n-1}(0)\right.\right.} \\
& \left.\left.-\left(T_{0,1} / T_{1,0}\right) p_{n-1}(1)\right]\left(\begin{array}{c}
1 \\
-1
\end{array}\right)\right\} \equiv\left(\begin{array}{c}
C_{1} \\
1-C_{1}
\end{array}\right)+C_{2}\left(\begin{array}{c}
1 \\
-1
\end{array}\right) .
\end{aligned}
$$

It is noted that all the elements of the transition matrix $T$

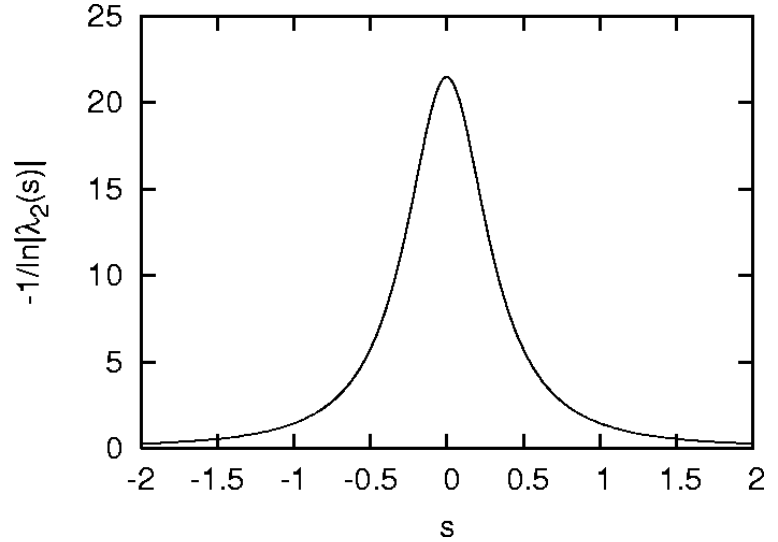

FIG. 2. $-1 / \ln \left|\lambda_{2}(s)\right|$ as a function of $s$ for $F=2$ and $\sigma_{\xi}=0.5$.

depend on time $n$ through the time dependence of the input signal $s_{n}$. The first and second vectors in Eq. (17) represent the equilibrium distribution $\boldsymbol{p}_{e q}\left(s_{n}\right)$ and the deviation therefrom.

By using the input signals, Eqs. (15), (16), and (17) we calculate $C_{1}$ (dotted curve) and $C_{2}$ (solid curve) [Fig. 4(a)]. It is noted that $C_{1}$, which is 1 (0) for small (large) $s$, depends only on $s$ but $C_{2}$ depends not only on $s$ but also on how input signals change with time (hysteresis effect). The arrows on the solid curve indicate the direction of time. We also show in Fig. 4(a) the eigenvalue $\lambda_{2}$ (curve with crosses). The average over a nonequilibrium distribution [8], $\langle y(n)\rangle=\sum_{y=0,1} y p_{n}(y)$, is shown in Fig. 4(b) together with $y_{e q}(s)=\sum_{y=0,1} y p_{e q}(y)$ (dotted curve) where $p_{e q}(y)$ is obtained by putting $C_{2}=0$ in Eq. (17) [see Eq. (12)].

If one takes the increasing signal, Eq. (15), the deviation $C_{2}$ starts to grow around $s=-1.3$, becomes largest at $s=-0.5$ where a vertical arrow is shown, and then goes to zero, recovering the equilibrium property for $s>1$. The largest deviation, also shown by a vertical arrow in Fig. 4(b), corresponds to $\langle y(s=-0.5)\rangle=0$ and $y_{\text {eq }}(s=-0.5)=1$. Similar behavior is observed also for the decreasing signals, Eq. (16).

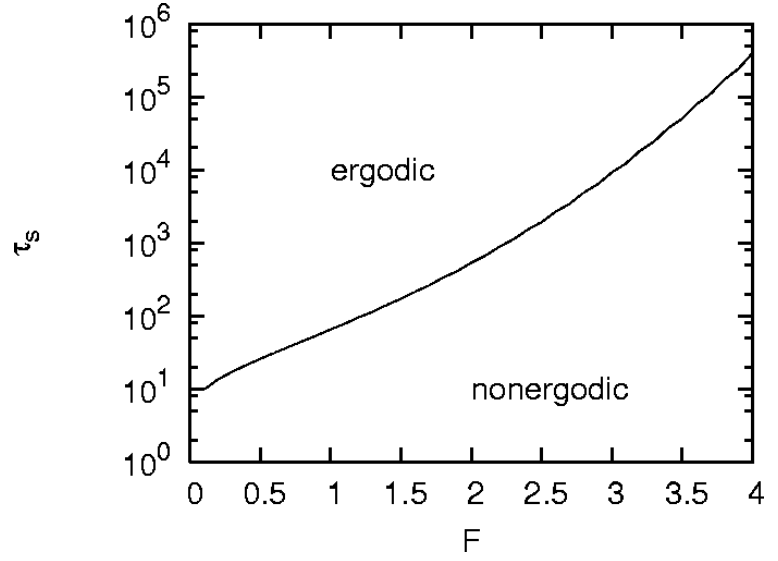

FIG. 3. $\tilde{\tau}_{S} \equiv \tau_{R} / \alpha$ as a function of $F$ (solid curve) for $\sigma_{\xi}=0.5$. Above (below) this curve $\left[\tau_{S}>(<) \tilde{\tau}_{S}\right]$ it is confirmed from numerical experiments that the system behaves as an ergodic (nonergodic) system. 


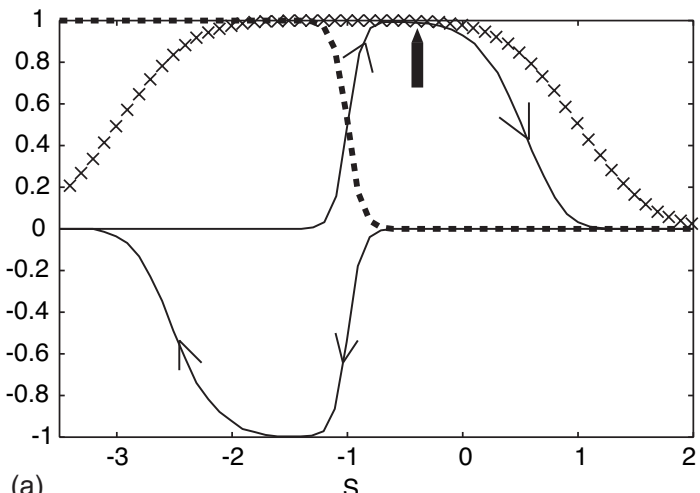

(a)

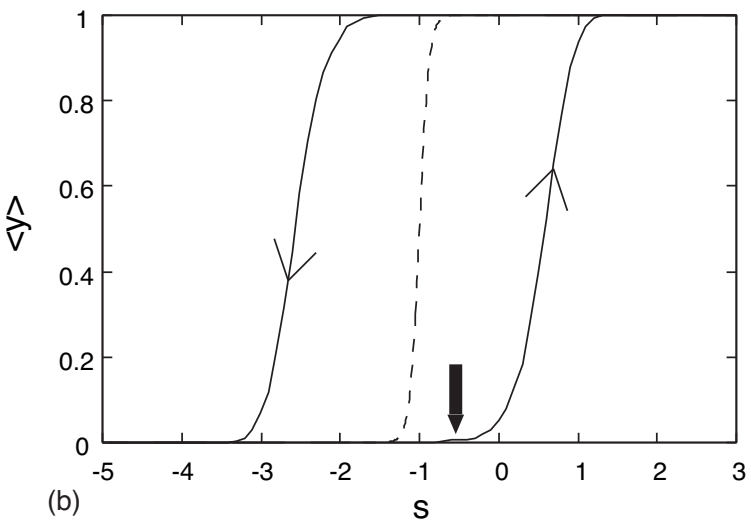

FIG. 4. (a): $\lambda_{2}(s)$ (curve with crosses) and $C_{1}(s)$ (dotted curve) are shown as functions of $s$ for $F=4, \tau_{S}=10^{2}$, and $\sigma_{\xi}=0.5$. The deviation $C_{2}$ in this case depends on the signal history and the arrows on the solid curves denote the direction of time. (b) The average over a nonequilibrium distribution, $\langle y\rangle$, for $s_{i n, n}$ and $s_{d e, n}$.

\section{MEAN-FIELD THEORY}

Up to now we have been paying attention to time evolution of the distribution function $\boldsymbol{p}_{n}$ and clarified the meaning of hystereses or ergodic-nonergodic transition in a simple threshold system with feedback, Eq. (3). From Eq. (6) with $\theta=1$ we immediately obtain

$$
\left\langle y_{n}\right\rangle=(1 / 2) \operatorname{erfc}\left[\left(1-s_{n}-F y_{n-1}\right) /\left(\sqrt{2} \sigma_{\xi}\right)\right] .
$$

In order to derive a self-consistent equation (SCE) from Eq. (18), we tentatively replace $y_{n-1}$ by $\left\langle y_{n-1}\right\rangle$, to be justified for a special (extended) model later in this section, and introduce an assumption that the input signals $s_{n}$ slowly change with $n$. Then one may write $\left\langle y_{n-1}\right\rangle \simeq\left\langle y_{n}\right\rangle$ and arrives at the SCE [4]

$$
\langle y\rangle_{s c}=(1 / 2) \operatorname{erfc}\left[\left(1-s-F\langle y\rangle_{s c}\right) /\left(\sqrt{2} \sigma_{\xi}\right)\right],
$$

which determines how the average $\langle y\rangle_{s c}$ depends on $F$ and $s$.

It is readily seen that Eq. (19) has a bifurcation point $F_{c}=\sigma_{\xi} \sqrt{2 \pi}$. That is, for $F<F_{c}$, the solution $\langle y\rangle_{s c}(s)$ is single valued [Fig. 5(a)] as a function of $s$, while for $F>F_{c},\langle y\rangle_{s c}(s)$ is multivalued in some region of $s, s_{0}<s<s_{1}$ [Fig. 5(b)]. By comparing $\langle y\rangle_{s c}(s)$ in Fig. 5(b) with $\lambda_{2}$ in Fig. 4(a), we notice that the multivalued region $s_{0}<s<s_{1}$ semiquantitatively corresponds to the region in $s$ where $\lambda_{2}$ is near 1. However, the SCE alone cannot clarify the meaning of the bifurcation
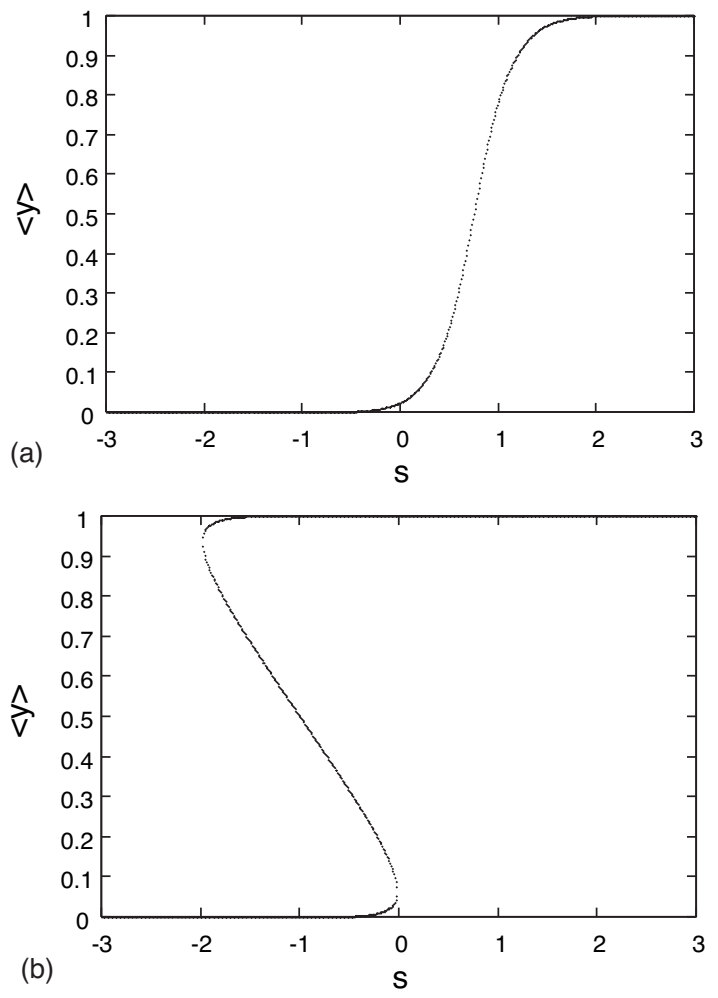

FIG. 5. Solution to the SCE (19) for $\sigma_{\xi}=0.5$ and $F=0.5$ (a) and $F=4($ b).

and multivaluedness of the solution of SCE. Our analyses based on the eigenvalues and eigenvectors in Secs. II and III clarified these points and revealed an ergodic-nonergodic transition.

To shed more light on the SCE (19), let us generalize the model, Eq. (3), by combining $M$ simple threshold systems in parallel (see Fig. 6) [9]. The output signal is defined by $y_{n} \equiv \sum_{1}^{M} y_{n}^{i} / M$, which can take the values $\{0,1 / M, \ldots, 1\}$. The transition probability is simply given for $y_{n}=y \equiv m / M$, and $y_{n-1}=y^{\prime} \equiv m^{\prime} / M$ by

$$
\begin{aligned}
p\left(y \mid y^{\prime}, s_{n}\right) & =\frac{M !}{m !(M-m) !} p\left(1 \mid y^{\prime}, s_{n}\right)^{m}\left[1-p\left(1 \mid y^{\prime}, s_{n}\right)\right]^{M-m} \\
& \equiv T_{y, y^{\prime}}\left(s_{n}\right)
\end{aligned}
$$

where $p\left(1 \mid y^{\prime}, s_{n}\right)$ is given by Eq. (6). The time evolution of the $(M+1)$-dimensional probability vector $p_{n}$ can be analyzed in a way quite similar to the one developed for the case $M=1$ in Sec. II. That is, the relaxation properties of this

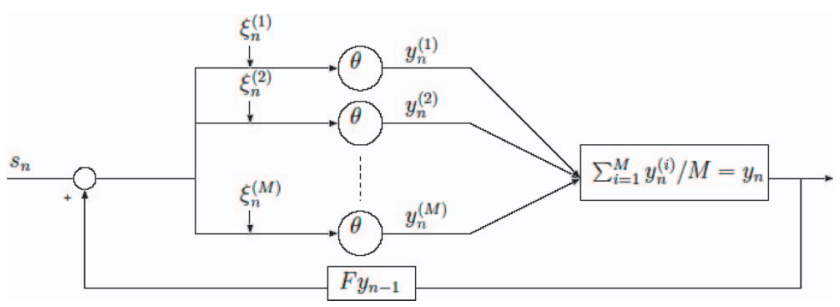

FIG. 6. $M$ units are combined to form a generalized system. 


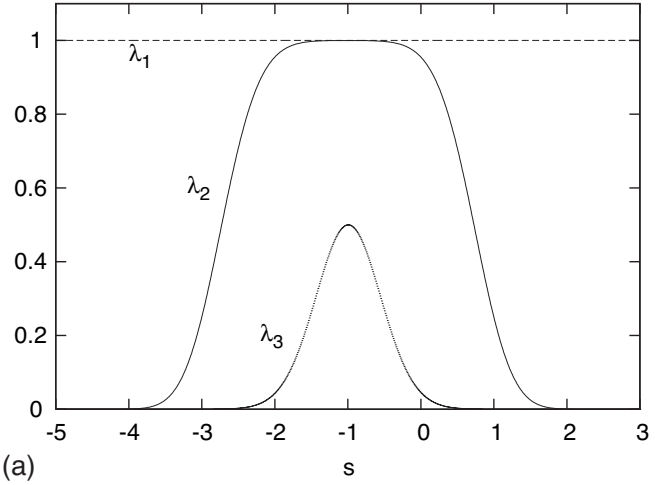

(a)

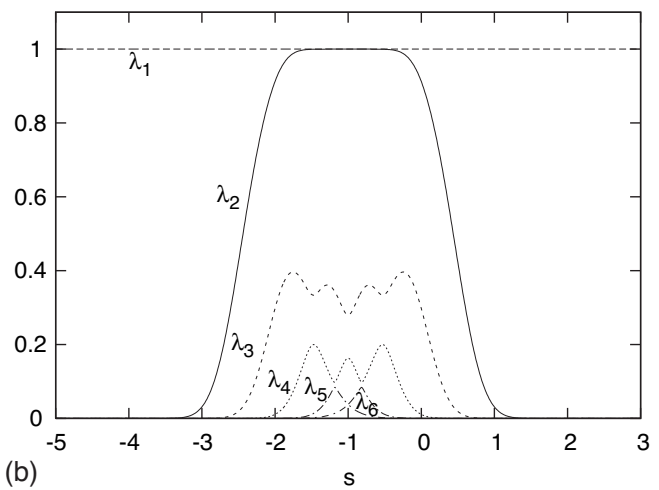

FIG. 7. Eigenvalues $\lambda_{1}(=1), \lambda_{2}, \ldots, \lambda_{M+1}$ are plotted as a function of $s$ for $M=2$ (a) and $M=5$ (b) for $F=4, \sigma_{\xi}=0.5$.

system can be investigated from the eigenvalues of the $(M+1) \times(M+1)$ matrix $T$. As examples, we show the eigenvalues $\left\{\lambda_{1}=1, \lambda_{2}, \ldots, \lambda_{M+1}\right\}$ put in a descending order for the cases $M=2$ and $M=5$ (Fig. 7). From other examples, also, it is seen that only $\lambda_{2}$ plays an important role for the ergodicnonergodic transition. In this sense we may say that additional complexity, except for the size of the matrix $T$, is not brought about by going to the system in Fig. 6 .

If one calculates the conditional mean $\left\langle y_{n} \mid y_{n-1}, s_{n}\right\rangle$ $\equiv \sum y_{n} p\left(y_{n} \mid y_{n-1}, s_{n}\right)$ from Eq. (20), we immediately have $\left\langle y_{n} \mid y_{n-1}, s_{n}\right\rangle=p\left(1 \mid y_{n-1}, s_{n}\right)$. Similarly we have, for the variance, $\sigma_{y_{n}}^{2}=p\left(1 \mid y_{n-1}, s_{n}\right)\left[1-p\left(1 \mid y_{n-1}, s_{n}\right)\right] / M$, which vanishes in the limit $M \rightarrow \infty$. From these facts it holds that $p\left(y \mid y^{\prime}, s_{n}\right)=\delta\left(y-p\left(1 \mid y^{\prime}, s_{n}\right)\right)$ as $M \rightarrow \infty$. In the same limit Eq. (7) is reduced to

$$
p_{n}(y)=\int_{0}^{1} d y^{\prime} p\left(y \mid y^{\prime}, s_{n}\right) p_{n-1}\left(y^{\prime}\right) .
$$

If we assume that input signals change slowly $\left(s_{n} \simeq s\right)$ and that $p_{n}(y)=\delta\left(y-y_{0}(s)\right)$ with $y_{0}(s)$ to be determined below, we have, from Eq. (21),

$$
\delta\left(y-y_{0}(s)\right)=\delta\left(y-p\left(1 \mid y_{0}(s), s\right)\right) .
$$

Equation (22) is equivalent to the SCE (22) and $y_{0}(s)=\langle y\rangle_{s c}(s)$. From the discussions above we know that the SCE (19) is valid only in the limit $M \rightarrow \infty$ where fluctuation effects can be neglected.

\section{COMMENT ON FEEDBACK STRENGTH AND CONCLUSION}

In this section we first try to interpret feedback strength $F$ using a conditional probability different from Eq. (6). For the purpose, we introduce the conditional probability

$$
p_{T}\left(y_{n}=1 \mid y_{n-1}, \tilde{s}_{n}\right) \equiv 1 /\left\{1+\exp \left[\left(\theta-\tilde{s}_{n}-2 F y_{n-1}\right) / T\right]\right\},
$$

characterized by $T$, to be called the temperature of the system. It is readily confirmed that Eq. (23) approximates Eq. (6) well if $T$ is chosen to be

$$
T=0.4 \sqrt{2} \sigma_{\xi}
$$

Hereafter we will employ Eq. (23), instead of Eq. (6), to make contact with statistical mechanics easier.

Introducing the transition probability by $W\left(0 \rightarrow 1 \mid \tilde{s}_{n}\right)$ $\equiv p\left(y_{n}=1 \mid y_{n-1}=0, \widetilde{s}_{n}\right)$ we have, from Eq. (23),

$$
\begin{gathered}
W\left(0 \rightarrow 1 \mid \tilde{s}_{n}\right)=1 /\left\{1+\exp \left[\left(\theta-\tilde{s}_{n}\right) / T\right]\right\}, \\
W\left(1 \rightarrow 0 \mid \widetilde{s}_{n}\right)=1 /\left\{1+\exp \left[\left(\tilde{s}_{n}+F-\theta\right) / T\right]\right\} .
\end{gathered}
$$

From Eqs. (10) and (25) we see that $\lambda_{2}\left(\tilde{s}_{n}^{i}\right)=W\left(0 \rightarrow 0 \mid \tilde{s}_{n}\right)$ $-W\left(1 \rightarrow 0 \mid \tilde{s}_{n}\right)$. It is easy to show, following the definition (13), that $\tau_{R}=\exp \left[F_{i} /(2 T)\right] / 2$ when $F$ is large. The exponential dependence of $\widetilde{\tau}_{S}$ on $F$ shown in Fig. 3 is qualitatively explained by this activation-type relaxation time.

In conclusion we studied some dynamical properties of a threshold system with feedback. As a by-product we also gave a comment on the relation between the feedback strength $F$ and an activation energy. By considering the time evolution of the distribution vector $\boldsymbol{p}_{n}$ instead of the average $\left\langle y_{n}\right\rangle$, we showed that the system, although simple, can have an ergodic-nonergodic transition. At this point it was also shown that the SCE is valid only in the limit $M \rightarrow \infty$. The interacting threshold model proposed in this section may be interesting from the point of a space-time-dependent threshold $\theta\left(x_{i}, n\right)(i=1,2, \ldots, M)$ with $x_{i}$ meaning the position of the $i$ th threshold system, where we expect a nontrivial interplay of both the feedback and the interaction.

\section{ACKNOWLEDGMENT}

This work was supported by a Grand-in-Aid from the Ministry of Education, Culture, Science and Technology of Japan. 
[1] J. Hertz, A. Krogh, and R. G. Palmer, Introduction to the Theory of Neural Computation (Addison-Wesley, 1991); S. Ishihara and K. Kaneko, Phys. Rev. Lett. 94, 058102 (2005).

[2] Y. Wang and L. Wu, Int. J. Signal Process. 2, 203 (2005), and references cited therein.

[3] L. Gammaitoni, Phys. Rev. E 52, 4691 (1995).

[4] T. Munakata, A. Sato, and T. Hada, J. Phys. Soc. Jpn. 74, 2098 (2005).

[5] L. Gammaitoni, P. Hänggi, P. Jung, and F. Marchesoni, Rev. Mod. Phys. 70, 223 (1998).

[6] V. S. Anishchenko, V. V. Astakhov, A. B. Neiman, T. E. Vadivasova, and L. Schimansky-Geier, Nonlinear Dynamics of Chaotic and Stochastic Systems (Springer, Berlin, 2002).

[7] In connection with the stochastic resonance and effects of feedback for double-well potential systems, see J. F. Lindner,
J. Mason, J. Neff, B. J. Breen, W. L. Ditto, and A. R. Bulsara, Phys. Rev. E 63, 041107 (2001); J. Mason et al., Phys. Lett. A 227, 13 (2000).

[8] Once the initial distribution $\boldsymbol{p}(n=0)$ and the signal sequence $s_{n}$ are chosen, the time evolution can be discussed based on $\left\langle y_{n}\right\rangle=\sum_{y=0,1} y p_{n}(y)$. This is equivalent to the average over many experiments performed under the same fixed conditions.

[9] A. H. Sato, M. Ueda, and T. Munakata, Phys. Rev. E 70, 021106 (2004).

[10] L. E. Reichl, A Modern Course in Statistical Physics (The University of Texas Press, Austin, 1980) Chap. 8.

[11] The Monte Carlo Method in Condensed Matter Physics, 2nd ed., edited by K. Binder (Springer, Berlin, 1997).

[12] K. Binder and D. W. Heermann, Monte Carlo Simulation in Statistical Physics, 2nd ed. (Springer, Berlin, 1997). 